NISTIR 7807

\title{
NIST Special Database 32 Multiple Encounter Dataset II (MEDS-II)
}

Data Description Document

\author{
Andrew P. Founds \\ Nick Orlans \\ Genevieve Whiddon \\ MITRE Corporation
}

Craig Watson

Information Technology Laboratory 
NISTIR 7807

\title{
NIST Special Database 32 Multiple Encounter Dataset II (MEDS-II)
}

Data Description Document

\author{
Andrew P. Founds \\ Nick Orlans \\ Genevieve Whiddon \\ MITRE Corporation \\ Craig Watson \\ Information Technology Laboratory \\ Information Access Division
}

February 2011

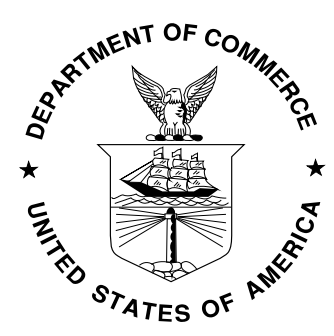

U.S. Department of Commerce

National Institute of Standards and Technology Patrick D. Gallagher, Deputy Director 


\section{Acknowledgement}

This dataset is being released (as prepared by MITRE Corporation) to support the NIST MultipleBiometric Evaluation 2010 (MBE). In addition, this dataset is available to any user interested in biometric research. The sponsor of this joint effort and provider of the data is the Federal Bureau of Investigation (FBI).

\section{Disclaimer}

Specific hardware and software products identified in this report were used in order to process and analyze the dataset described in this document. In no case does identification of any commercial product, trade name, or vendor, imply recommendation or endorsement by the National Institute of Standards and Technology, nor does it imply that the products and equipment identified are necessarily the best available for the purpose. 


\title{
Data Description Document
}

\author{
Andrew P. Founds \\ Nick Orlans \\ Genevieve Whiddon \\ Version 4.0 \\ December 22, 2010
}





\section{MITRE}

Sponsor: FBI CJIS

Dept. No.: G123

Contract No.: J-FBI-07-164

Project No.: 1410FC09

Downgrade UNCLASSIFIED

Derived By:

Declassify On:

The views, opinions and/or findings contained in this report are those of The MITRE Corporation

and should not be construed as an official government position, policy, or decision, unless designated by other documentation.

This technical data was produced for the U.S. Government under contract JFBI-07-164 and is subject to the Rights in Data-General Clause 52.227-14, Alt. IV (JUNE 1987).

This document was prepared for authorized distribution only. It has not been approved for public release.

(C)2010 The MITRE Corporation. All Rights Reserved.

\section{Multiple Encounter Dataset (Deceased Persons) MEDS-II}

\author{
Data Description Document
}

\author{
Andrew P. Founds \\ Nick Orlans \\ Genevieve Whiddon
}

Version 4.0

December 22, 2010 



\section{Table of Contents}

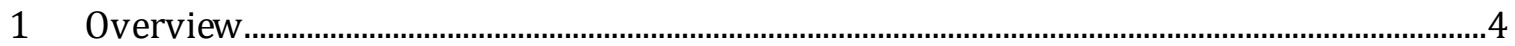

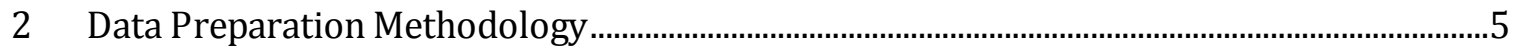

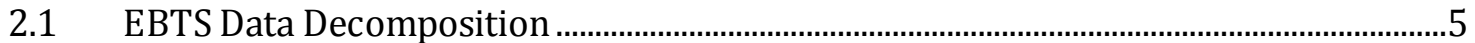

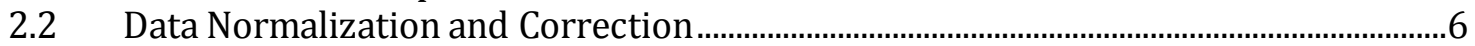

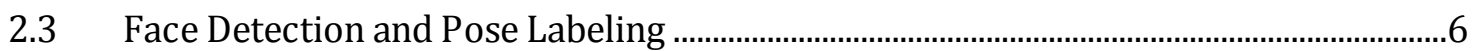

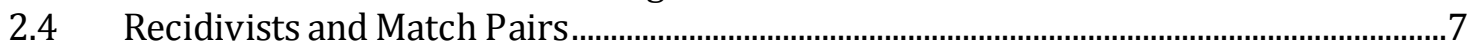

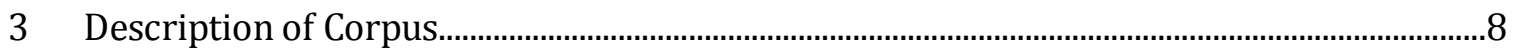

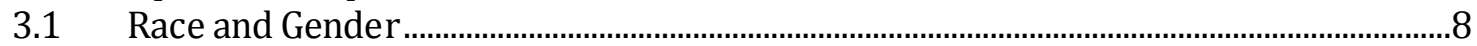

3.2 Age Summary and Time between Encounters .................................................................

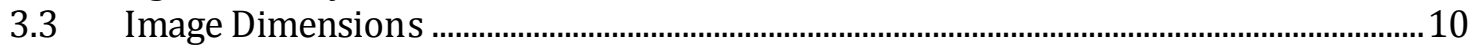

3.4 Face Resolution and Subject Pose

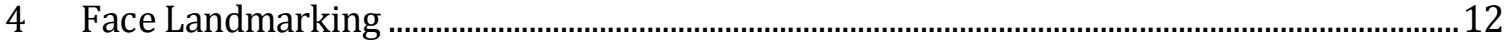

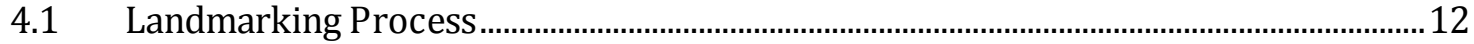

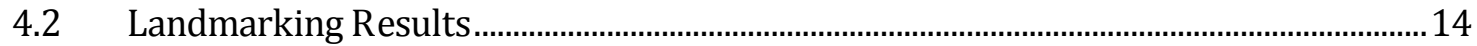

4.3 Next Steps for Landmarking and the MEDS-II Corpus.......................................................16

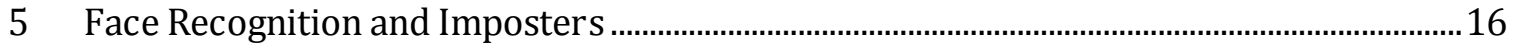

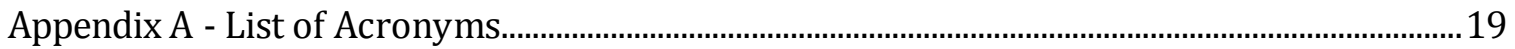




\section{List of Figures}

Figure 1 - Relationship between Subjects, Submissions and Biometric Samples .......................6

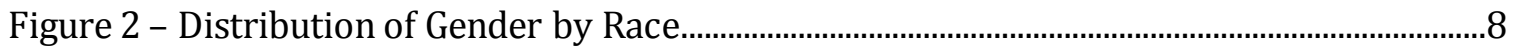

Figure 3 - Histogram of Subject Ages at Photo Date ..............................................................................9

Figure 4 - Times between Encounters (e.g., first and last) ..................................................................

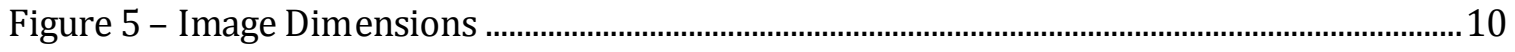

Figure 6 - Distribution of Estimated Interocular Distances (in pixels) .........................................11

Figure 7 - Face Roll Angle (degrees from horizontal) ..........................................................................11

Figure 8 - Example Stasm Outputs ……............................................................................................ 12

Figure 9 - Depiction of 68 Stasm Points.............................................................................................. 13

Figure 10 - Proportion of Automated vs. Manual Landmarking .......................................................14

Figure 11 - Ability to Automatically Landmark Based on Race ........................................................14

Figure 12 - Ability to Automatically Landmark by Gender ................................................................15

Figure 13 - Correlation of PittPatt Confidence Scores with Automated Landmarking ............15

Figure 14 - Correlation of PittPatt Yaw Estimate with Automated Landmarking.....................15

\section{List of Tables}

Table 1 - MEDS-II Dataset Overview ……………............................................................................

Table 2 - Tools used to parse and examine sub mission files .............................................................5

Table 3 - Number of Type-10 Images and Submissions ...................................................................6

Table 4 - Image Types ......................................................................................................................

Table 5 - Enumeration of Match Pairs ................................................................................................

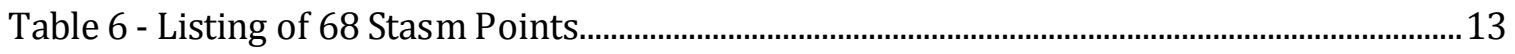

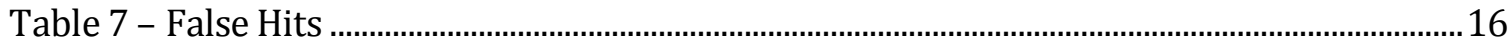

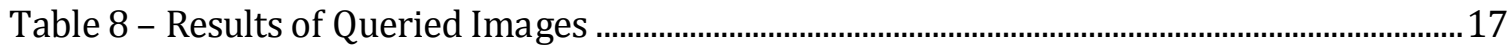




\section{Overview}

This document and associated dataset is an update to the Multiple Encounter Dataset I (MEDS-I), originally published by the National Institute of Standards and Technology (NIST) in May 2010 ${ }^{1}$. The MEDS is a test corpus organized from an extract of submission files of deceased persons with prior multiple encounters. A submission file is an electronic file containing biographic and biometric data recorded during an encounter of an individual. The submission files conform to the specifications defined by the Electronic Biometric Transmission Specification (EBTS) extension to the American National Standards Institute (ANSI)/NIST Information Technology Laboratory (ITL)-1-2007 standard2.

MEDS-I and MEDS-II are intended to stimulate research and to assist with the NIST Multiple Biometric Evaluation. The MEDS-II update approximately doubles the number of images, and extends the metadata to better support research and evaluation on pose conformance and local face features. These data are provided to assist the FBI and partner organizations refine tools, techniques, and procedures for face recognition as it supports Next Generation Identification (NGI), forensic comparison, training, analysis, and face image conformance and inter-agency exchange standards. The MITRE Corporation (MITRE) prepared MEDS-I and MEDS-II in the FBI Data Analysis Support Laboratory (DASL).

This paper describes the basic properties of the images and some relevant image quality characteristics that pertain to collection practices and the calibration and evaluation of face recognition technology. Table 1 provides an overview of the final contents of the MEDS-I and MEDS-II corpus.

Table 1 - MEDS-II Dataset Overview

\begin{tabular}{|l|c|c|c|}
\hline \multicolumn{1}{|c|}{ Dataset } & $\begin{array}{c}\text { Subject } \\
\text { Count }\end{array}$ & $\begin{array}{c}\text { Submission } \\
\text { Count }\end{array}$ & $\begin{array}{c}\text { Image } \\
\text { Count }\end{array}$ \\
\hline MEDS-I & 380 & 682 & 711 \\
\hline MEDS-II & 138 & 535 & 598 \\
\hline MEDS-I \& MEDS-II & 518 & 1,217 & 1,309 \\
\hline
\end{tabular}

All original submissions contain at least one logical Type-10 record, the record type within the ANSI/NIST-ITL 1-2007 file format reserved for face images and Scars, Marks, and Tattoos (SMT) images. The submission files were parsed into the various record types, as described below in Section 2.

\footnotetext{
${ }^{1}$ Watson, C. I. (2010, May 10). NIST Special Database 32 - Multiple Encounter Dataset I (MEDS-I). Retrieved December 13, 2010, from National Institutes of Standards and Technology: http://www.nist.gov/itl/iad/ig/sd32.cfm

${ }^{2}$ American National Standard for Information Systems - Data Format for the Interchange of Fingerprint, Facial, and other Biometric Information - Part 1. NIST Special Publication 500-271, May, 2007. Online: http://fingerprint.nist.gov/standard/Approved-Std-20070427.pdf
} 


\section{Data Preparation Methodology}

This section describes the processes of EBTS decomposition, data normalization and correction, and face detection necessary to prepare this corpus.

\subsection{EBTS Data Decomposition}

The submission files were parsed and examined using a combination of government, commercial, and custom EBTS parsing and reporting tools to help verify consistent results. Table 2 presents a summary of tools used.

Table 2 - Tools used to parse and examine submission files

\begin{tabular}{|l|l|l|l|}
\hline \multicolumn{1}{|c|}{ Tool } & \multicolumn{1}{c|}{ License } & \multicolumn{1}{c|}{ Developer } & \multicolumn{1}{c|}{ Purpose } \\
\hline $\begin{array}{l}\text { Universal Latent } \\
\text { Workstation }\end{array}$ & GOTS & Noblis ${ }^{3}$ & Manual EBTS inspection \\
\hline EFTSExtract & GOTS & MITRE & Batch extraction and reporting \\
\hline Google Picasa & COTS & Google & Gallery viewing \\
\hline PittPatt 4 & COTS & $\begin{array}{l}\text { Pittsburgh Pattern } \\
\text { Recognition }\end{array}$ & Tools for face detection \\
\hline Stasm & N/A & $\begin{array}{l}\text { S. Milborrow, F. } \\
\text { Nicolls }\end{array}$ & $\begin{array}{l}\text { Annotation of face contours and } \\
\text { features }\end{array}$ \\
\hline MarkIt & GOTS & MITRE & $\begin{array}{l}\text { Face annotation and point } \\
\text { editing }\end{array}$ \\
\hline matplotlib & PSF & J. Hunter $^{7}$ & Data visualization \\
\hline
\end{tabular}

Each submission file contained an associated subject identifier to indicate the link between a subject and their encounters (i.e., submission files or recording events) over time. For many subjects in the set, more than one submission file was provided. Multiple encounters of individuals are sometimes referred to as recidivist encounters. The time interval between multiple encounters varies per individual. The cardinal relationship between subjects and submissions and samples is shown in Figure 1.

\footnotetext{
${ }^{3}$ http://www.noblis.org

${ }^{4}$ http://pittpatt.com

${ }^{5}$ Milborrow, S., \& Nicolls, F. (2008). Locating Facial Features with an Extended Active Shape Model. ECCV, http://www.milbo.users.sonic.net/stasm.

${ }^{6}$ Pruitt, M. (1, June 2010). MarkIt. McLean, VA, USA

${ }^{7}$ Hunter, J. (2010, November 9). matplotlib Release 1.o.o. Retrieved December 13, 2010, from matplotlib: http://matplotlib.sourceforge.net/index.html
} 


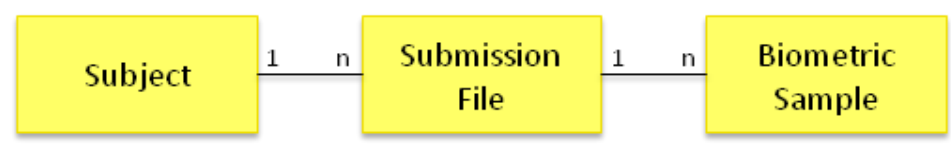

Figure 1 - Relationship between Subjects, Submissions and Biometric Samples

After establishing ground truth for this dataset (described in Section 3), the Type-10 images were assessed for their face content. Table 3 summarizes the image content as observed in the original submission files. Not all images are face images or considered part of the MEDSII dataset.

Table 3 - Number of Type-10 Images and Submissions

\begin{tabular}{|c|c|l|}
\hline $\begin{array}{c}\text { Number of } \\
\text { Images }\end{array}$ & $\begin{array}{c}\text { Number of } \\
\text { Submissions }\end{array}$ & Comments \\
\hline 1 & 1,217 & 1st image is frontal or near frontal face image \\
\hline 2 & 72 & $2^{\text {nd }}$ image is usually a profile face image \\
\hline 3 & 20 & $3^{\text {rd }}$ image is usually a profile face image \\
\hline
\end{tabular}

\subsection{Data Normalization and Correction}

The consistency and reliability of the biographic data in the submissions varies, presumably due to input error or inconsistent information collection from subjects who may not have cooperated with the process. Some data normalization and corrections were performed to alleviate these errors on the metadata relevant to face detection and recognition (e.g., dates, gender, and race fields).

The date of arrest (DOA) and photo date (PHD) should be, by definition, within close date proximity of each other, and the PHD should always follow the DOA if the dates are not identical. In instances where either of these dates was missing or corrupt, the most repeated date among the entries was used for analysis. In the accompanying metadata file, an indicator is used to identify which records had been modified from their original contents.

\subsection{Face Detection and Pose Labeling}

Executing automated face detection was the first step in distinguishing the face-containing images from non-face-containing images. The face-containing images were additionally delineated into frontal and non-frontal bins based on the PittPatt (the tool used for face detection) pose estimates. Human reviewers manually reviewed each category to remove residual errors and obtain the final ground truth for the subject's pose.

As shown in Table 4, below, 1,219 of the images are frontal or "near frontal", as determined by human review. Frontal images are defined as within 15 degrees horizontal of full frontal, as estimated by visual inspection. Near frontal is defined as within 45 degrees horizontal, 
but not overlapping with the defined yaw angle range for frontal. Profiles or "near profiles" are likewise within 45 degrees of full profile, although "two-eyed" near profiles may be closer to 45-60 degrees off full profile. These definitions are working definitions and are prone to human error. Images where the pose yaw angle is compounded with pitch and roll deviations are even more prone to human review variations.

Table 4 - Image Types

\begin{tabular}{|c|c|c|}
\hline Type & Count & Comments \\
\hline Frontal & 858 & $\approx[-15,15]$ degrees yaw angle \\
\hline Near Frontal & 361 & $\approx[-45,-15) \cup(15,45]$ degrees yaw angle \\
\hline Near Profile & 6 & $\approx(-60,-45) \cup(45,60)$ degrees yaw angle \\
\hline Profile & 85 & $\approx[-90,-60] \cup[60,90]$ degrees yaw angle \\
\hline Total: & 1,309 & \\
\hline
\end{tabular}

\subsection{Recidivists and Match Pairs}

Near profile and profile images are included in the dataset to benefit research and development; however, these images are omitted in the count of match pairs. After profile and near profile images were removed, the number of match pairs is based on the remaining frontal or near frontal images (1,219 images). Table 5 enumerates the number of match pairs over the subjects based on the number of images per subject. The table only refers to frontal and near-frontal images.

Table 5 - Enumeration of Match Pairs

\begin{tabular}{|c|c|c|}
\hline $\begin{array}{c}\text { Number of } \\
\text { Subjects }\end{array}$ & $\begin{array}{c}\text { Number of } \\
\text { Images }\end{array}$ & $\begin{array}{c}\text { Number of } \\
\text { Match Pairs }\end{array}$ \\
\hline 262 & 1 & N/A \\
\hline 124 & 2 & 124 \\
\hline 47 & 3 & 141 \\
\hline 22 & 4 & 132 \\
\hline 15 & 5 & 150 \\
\hline 12 & 6 & 180 \\
\hline 9 & 7 & 189 \\
\hline 9 & 8 & 252 \\
\hline 4 & 9 & 144 \\
\hline 6 & 10 & 270 \\
\hline 5 & 11 & 275 \\
\hline 1 & 13 & 78 \\
\hline 1 & 16 & 120 \\
\hline 1 & 18 & 153 \\
\hline & Total: & 2,208 \\
\hline & & \\
\hline
\end{tabular}




\section{Description of Corpus}

This section provides a summary of the subject metadata contained within the MEDS-II dataset.

\subsection{Race and Gender}

Race and gender information are bas ed on observation or provided by the subject. Race can be ambiguous and ultimately is a social or cultural interpretation (as opposed to a consistently defined attribute for labeling). Race and gender, as provided in the data, is shown in Figure 2.

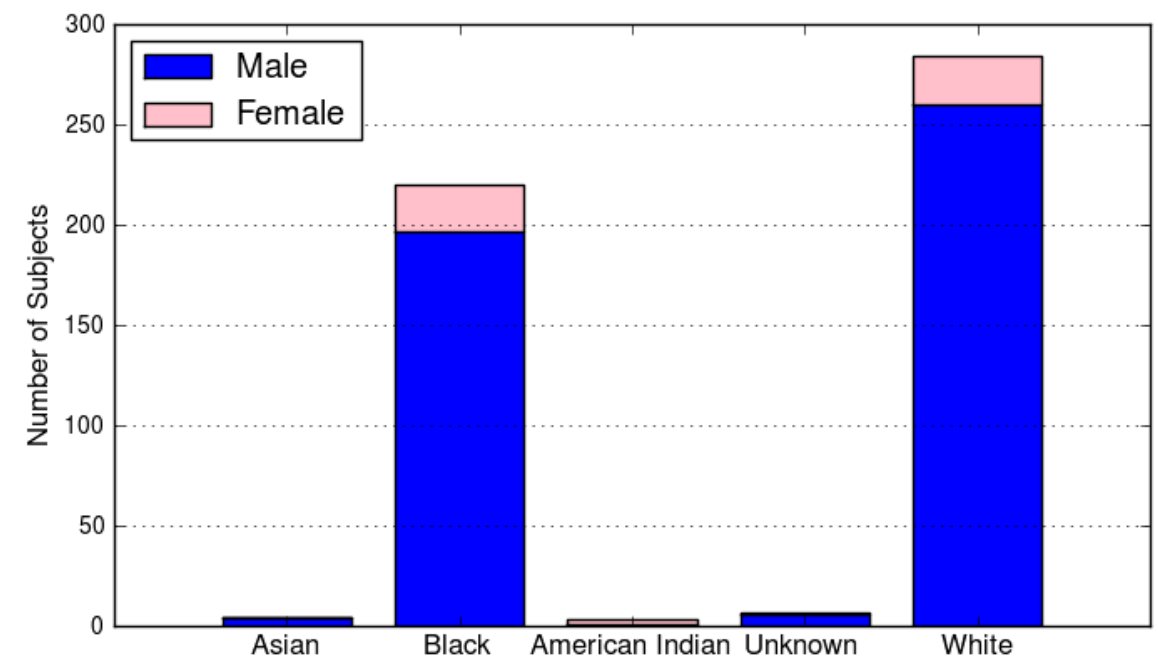

Figure 2 - Distribution of Gender by Race

\subsection{Age Summary and Time between Encounters}

Figure 3, below, illustrates the ages of the 518 subjects at the time the images were captured. The age of the subjects at the time of collection is also provided in the accompanying metadata for this dataset. 


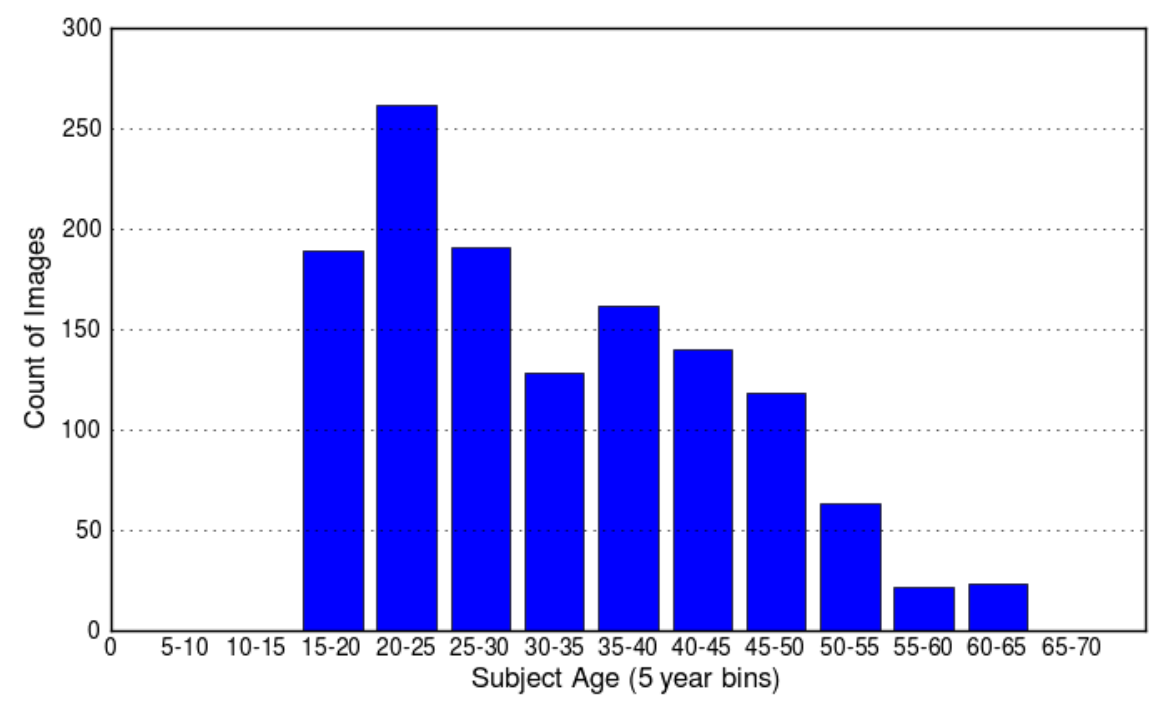

Figure 3 - Histogram of Subject Ages at Ph oto Date

Of the images in the dataset, $48 \%$ are of subjects between the ages of 15 and 30 years of age. Nine percent of the images in the dataset are of subjects greater than 50 years old while the oldest subject in the dataset is 69 years of age.

Figure 4 illustrates the times between the first and last encounter for all the subjects with multiple encounters. The horizontal axis is organized in bins of six month intervals.

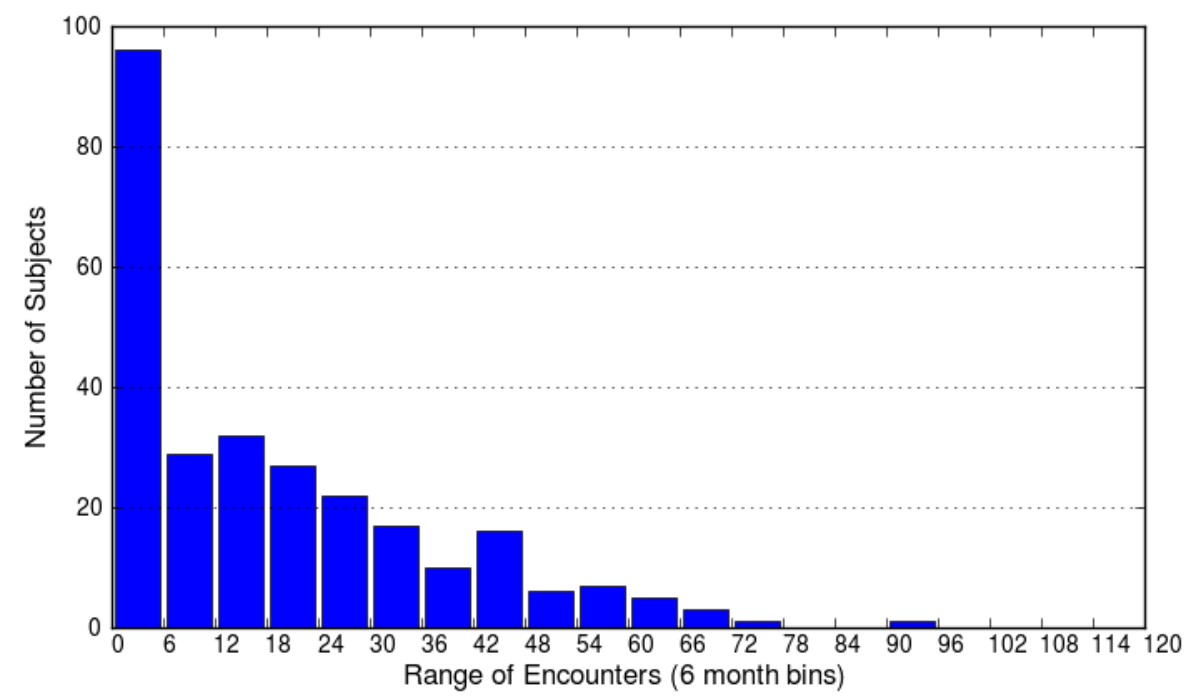

Figure 4 - Times between Encounters (e.g., first and last) 
Of the times between encounters, $47 \%$ are less than one year. The remainders are between one and five years (49\%) and greater than five years (4\%).

\subsection{Image Dimensions}

Image sizes and approximate resolution of the face vary due to the use of different camera equipment and composition inconsistencies of the subject in the image frame. Figure 5 is comprised of three charts, a histogram which illustrates the number of images by width, a histogram which illustrates the number of images by height, and a scatter plot which illustrates the number of images by both height and width. Of the images' dimensions, 70\% are approximately 0.3 megapixels while one image exceeds five megapixels. The red box in Figure 5 identifies the dimensions of roughly $70 \%$ of all images in the corpus.
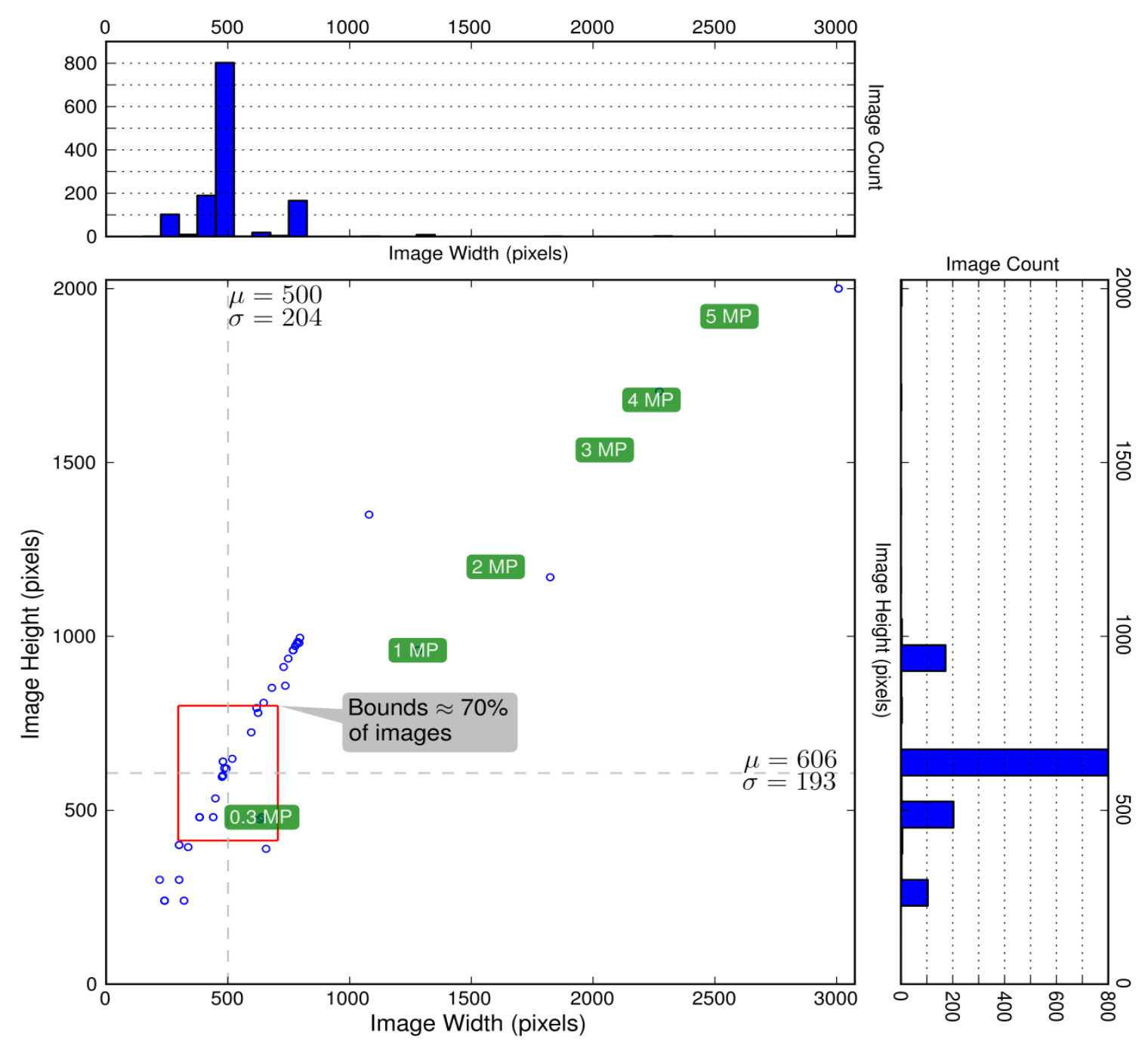

Figure 5 - Image Dimensions 
According to section 6 of the current specification, the ITL has no image scanning resolution for Type-10 records: "Facial/mugshot, SMT, and iris images rely on the total number of pixels scanned and transmitted and are not dependent on the specific scanning resolution used."

\subsection{Face Resolution and Subject Pose}

Consistent face resolution requires consistent sizing and framing. The framing of the subjects in the MEDS-I and MEDS-II images varies and, in some instances, the full face is not visible. For the frontal and near frontal images, MITRE estimated interocular distances based on the outputs from automated face detection. The results presented in Figure 6 and Figure 7 are based on 1,219 images that have been identified as frontal or near frontal andare based on automated outputs that were not reviewed or adjusted by human review.

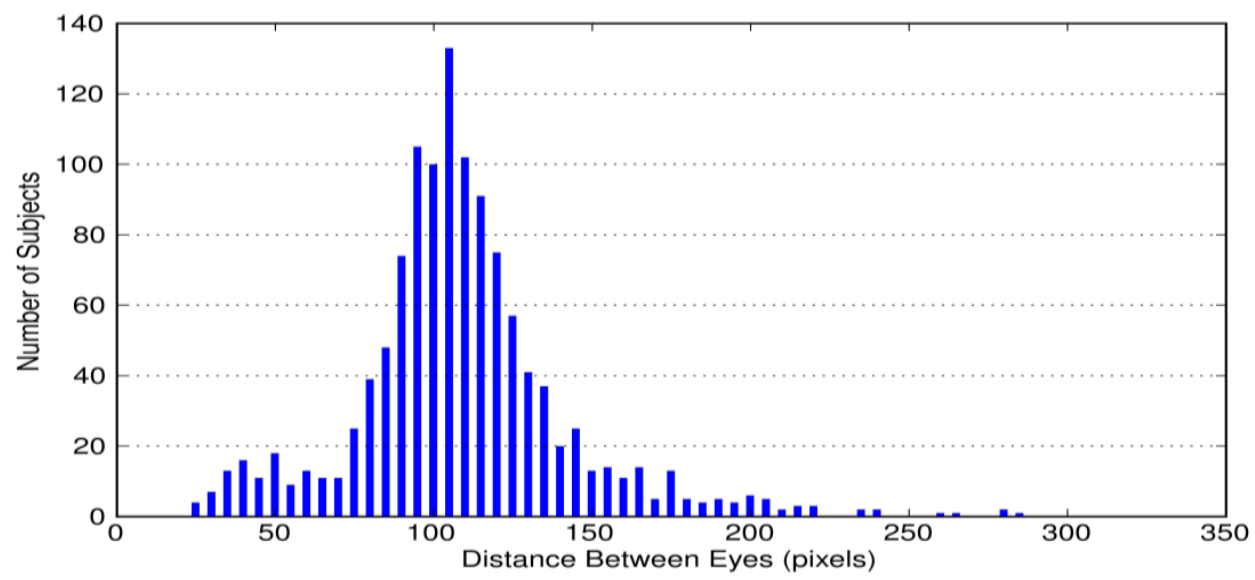

Figure 6 - Distribution of Estimated Interocular Distances (in pixels)

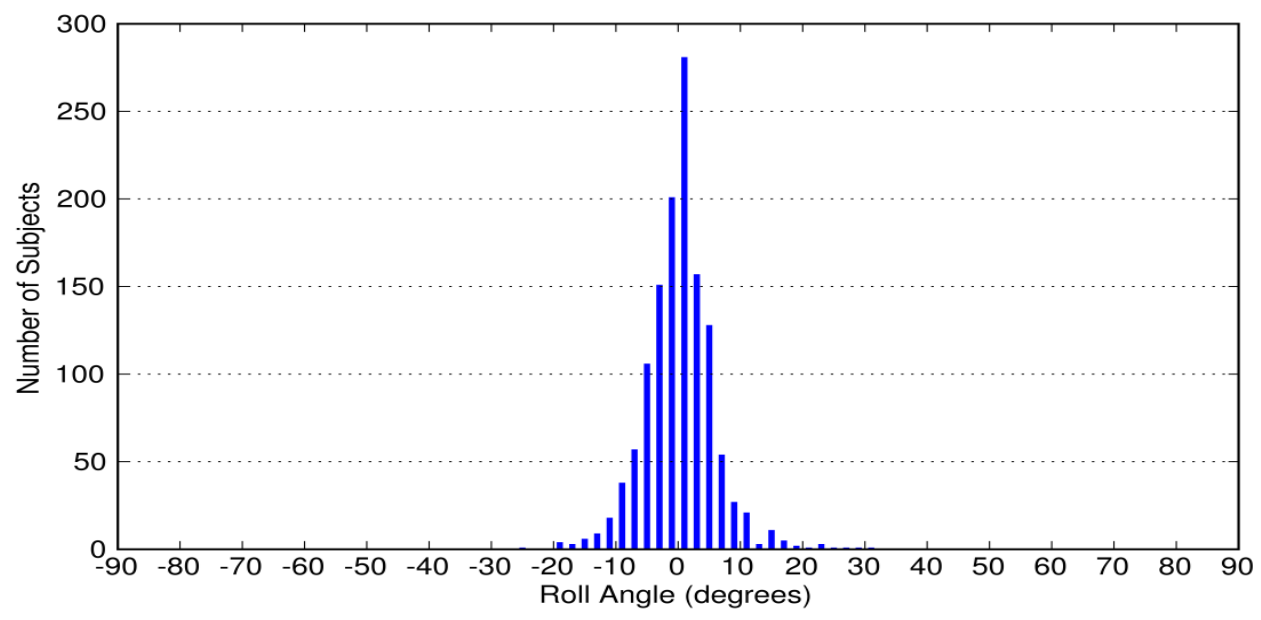

Figure 7 - Face Roll Angle (degrees from horizontal) 


\section{Face Landmarking}

\subsection{Landmarking Process}

Accurate landmarking of face images indicates successful localization of facial features and also may help with determining pose estimation and conformance. The MEDS-II images include a set of facial landmarks output by Stasm, an automated face landmarking tool based on Active Shape Models (ASM). Stasm is designed to work on passport-style photographs or on frontal views with neutral expressions.

Although all MEDS-II images were processed using Stasm, a portion of the images required manual correction in cases where the Stasm points were deemed inaccurate. As with most computer vision techniques, Stasm's ability to locate face landmarks is not as accurate as a human, and will occasionally make errors. In certain circumstances, manual editing of Stasm points was done with a custom tool, MarkIt, developed for face landmarking. Based on MITRE's empirical evidence, those images that exemplify poor lighting or extreme subject expressions tend to contain numerous errors. Cropped images will have unusable or stray points. Among images that had to be annotated manually, the contour of the jaw line proved to be a predominantly difficult area for Stasm, particularly if the subject had a beard or the contour of the jaw line was of low contrast. Some low contrast images were observed to improve performance after the contrast was boosted; however additional analysis is required. Figure 8 shows examples of images with output Stasm points overlaid on the image.
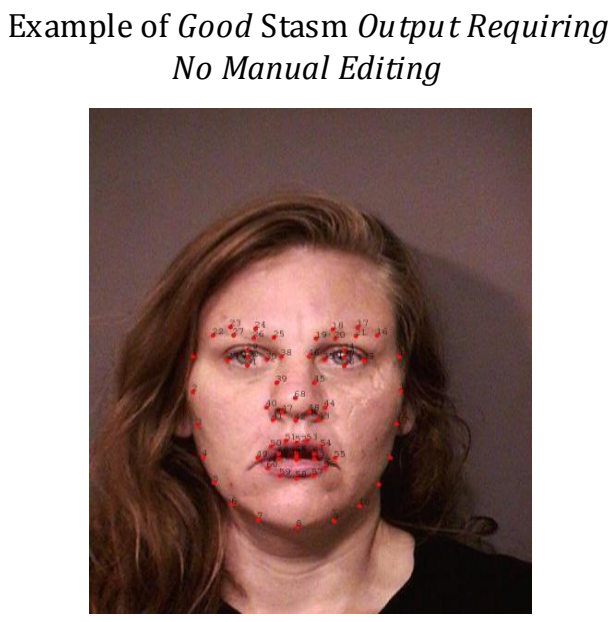

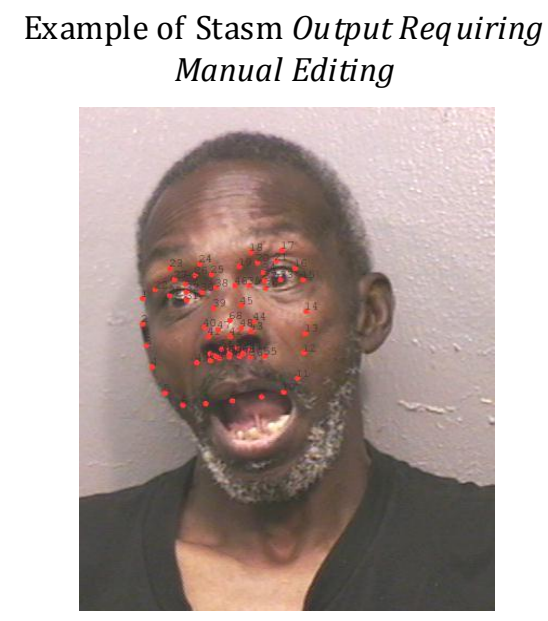

Figure 8 - Example Stasm Outputs

Stasm outputs a total of 68 points which correspond to an $(x, y)$ pixel value in the image. Each point corresponds to a unique facial landmark. These points are depicted in Figure 9 and enumerated in Table 6. 


\section{Multiple Encounter Dataset (MEDS-II)}

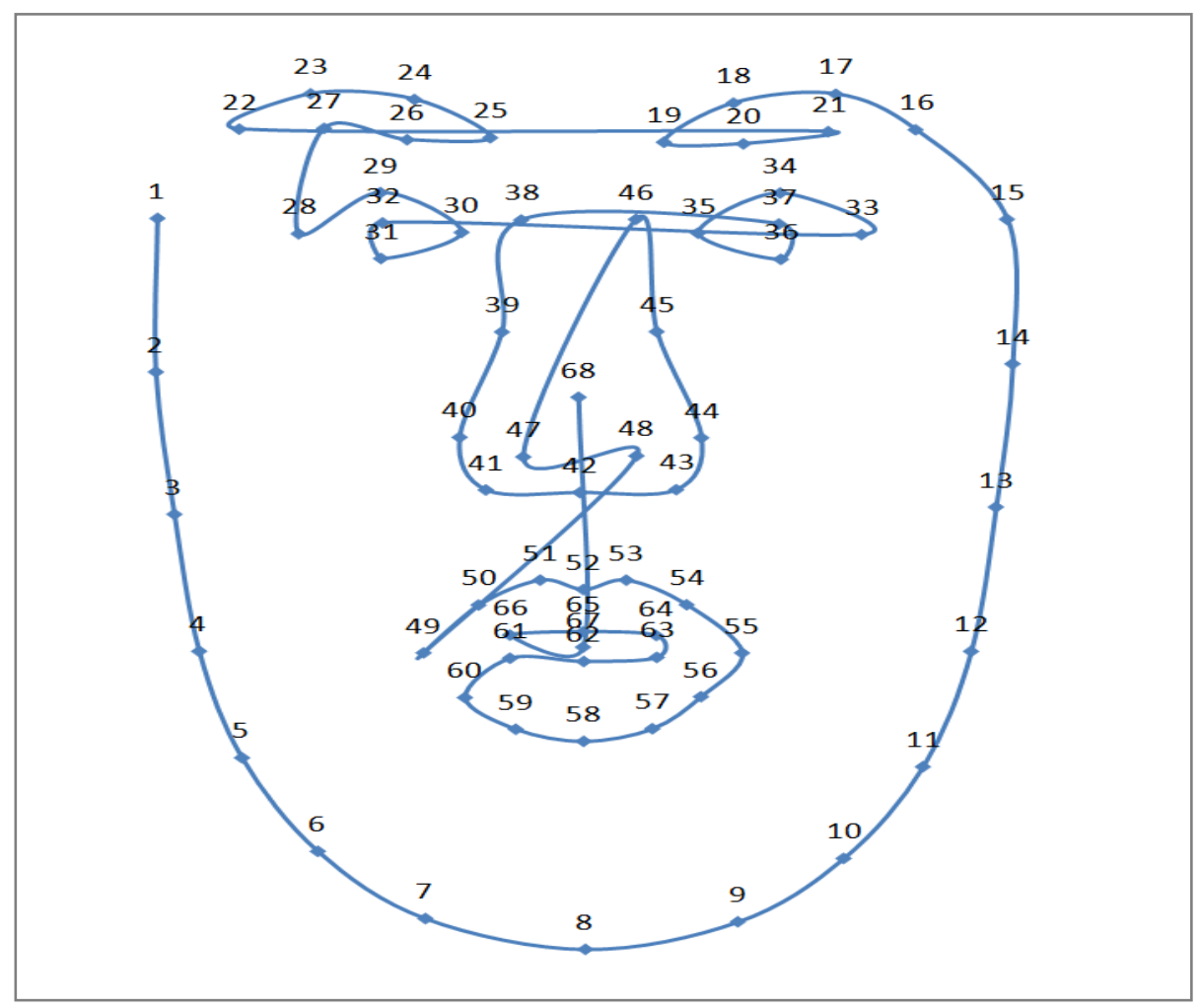

Figure 9 - Depiction of 68 Stasm Points

Table 6 - Listing of 68 Stasm Points
1. Right Temple
18. Left EyebrowInner Top
35. Left Eye Inner
52. Lip Top
2. Right Zygion
19. Left Eyebrow Inner
20. Left Eyebrow Inner Bottom
36. Left Eye Bottom
53. Left Lip Inner Top
3. Right Cheek Top
37. Left Pupil
54. Left Lip Outer Top
4. Right Cheek Bottom
21. Left Eyebrow Outer Bottom
38. Right Nasion
55. Left Chelion
5. Right Gonion
22. Right Eyebrow Outer
39. Right Alare Crease
56. Left Lip Outer Bottom
6. Right Chin Top
23. Right Eyebrow Outer Top
7. Right Chin Bottom
24. Right Eyebrow Inner Top
40. Right Alare
57. Left Lip Inner Bottom
8. Menton
25. Right Eyebrow Inner
41. Right Nostril
58. Lip Bottom
9. Left Chin Bottom
26. Right Eyebrow Inner Bottom
42. Subnasale
59. Right Lip Inner Bottom
10. Left Chin Top
27. Right Eyebrow Outer Bottom
43. Left Nostril
60. Right Lip Outer Bottom
11. Left Gonion
28. Right Eye Outer
44. Left Alare
61. Right Lip Bottom Center
12. Left Cheek Bottom
29. Right Eye Top
45. Left Alare Crease
62. Bottom Stomion
13. Left Cheek Top
30. Right Eye Inner
46. Left Nasion
63. Left Lip Bottom Center
14. Left Zygion
31. Right Eye Bottom
15. Left Temple
32. Right Pupil
33. Left Eye Outer
47. Right Nose Tip
64. Left Lip Top Center
48. Left Nose Tip
65. Top Stomion
49. Right Chelion
66. Right Lip Top Center
16. Left Eyebrow Outer
34. Left Eye Top
50. Right Lip Outer Top
67. Stomion
17. Left Eyebrow Outer Top
68. Pronasale 


\subsection{Landmarking Results}

Ninety-two percent (1,226 images) of the MEDS corpus was processed using the Stasm tool. In cases where the image was determined to be a profile, fingerprint, or marking (e.g., scar or tattoo), Stasm was not used and no landmark locations were generated. All Stasm result points were normalized according to the width and height of the image in pixels and subsequently compiled for analysis.

Nearly eighty percent of the images processed by the Stasm tool were considered acceptable by human analysis. In some cases, output could not be produced due to the tool's inability to detect a face. A summary of the percentage of images able to be processed by Stasm is shown in Figure 10.

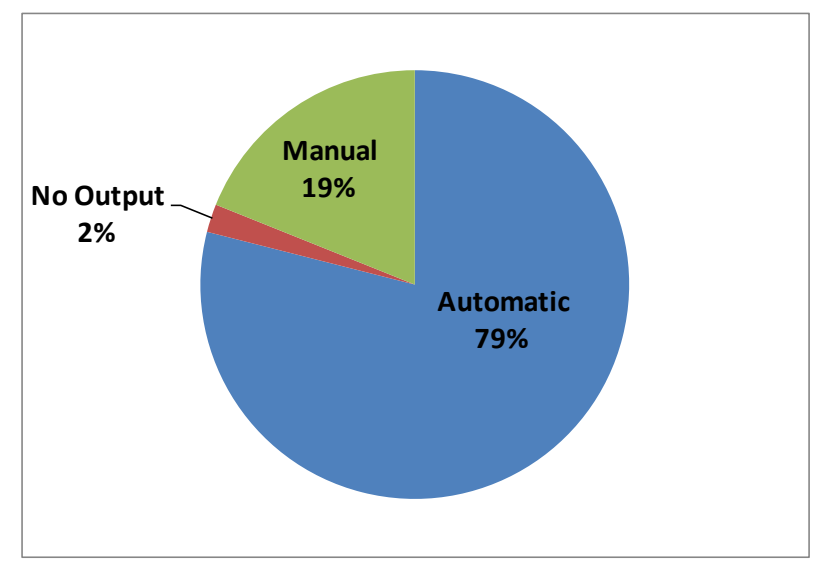

Figure 10 - Proportion of Automated vs. Manual Landmarking

Figure 11 and Figure 12 further isolate Stasm's ability to produce automated land marks based on race and gender, respectively. MITRE postulates that the reason for performing a higher percentage of manual landmarking on Black or African American subjects is due to the lack of contrast between chin and neck in the image. Additionally, MITRE hypothesizes that male images having beards failed automated landmarking.

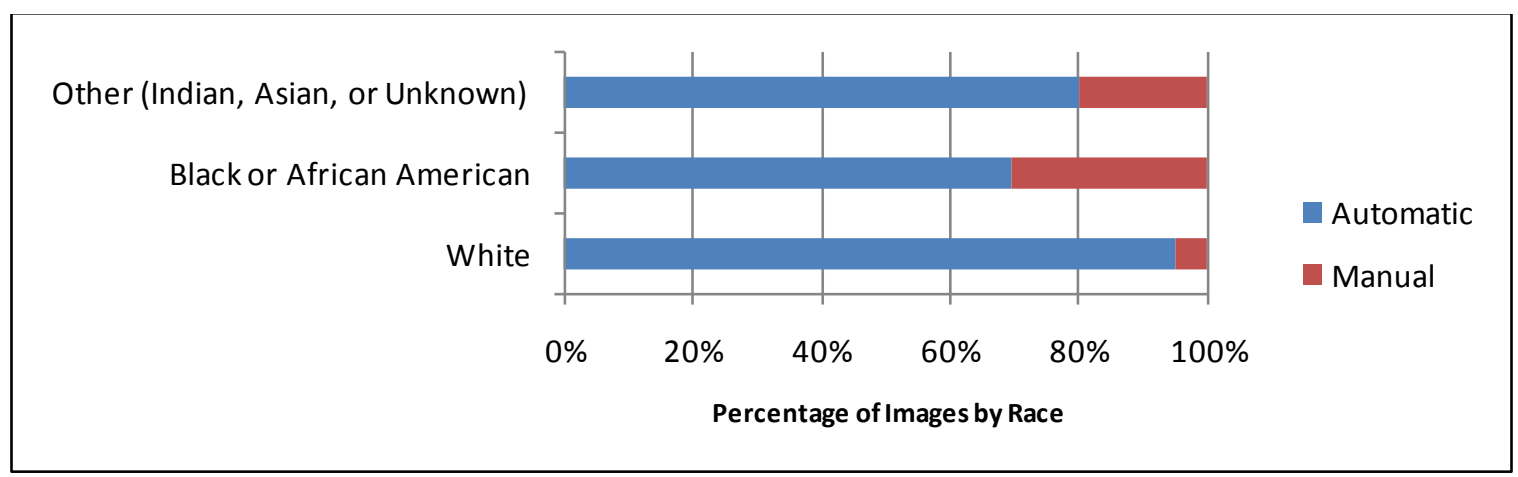

Figure 11 - Ability to Automatically Landmark Based on Race 


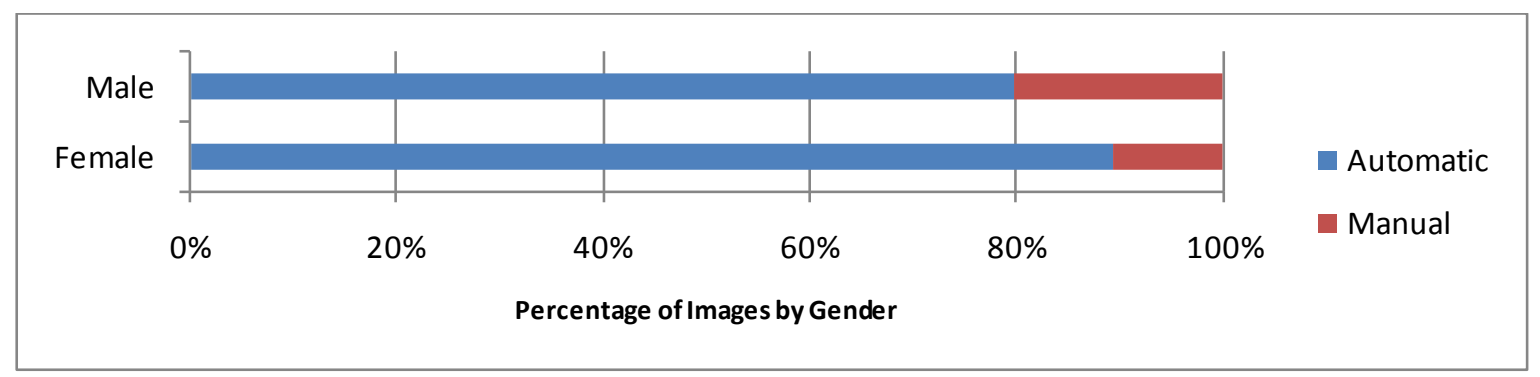

Figure 12 - Ability to Automatically Landmark by Gender

A high level analysis between PittPatt's confidence scores and yaw values, as correlated with Stasm output (e.g., rejection or acceptance by a human reviewer) was completed. Figure 13 and Figure 14 depict the correlation of confidence scores and yaw values, respectively, against Stasm's ability to successfully landmark an image. The impact appears to be minimal with little correlation between a confidence scores and yaw values.

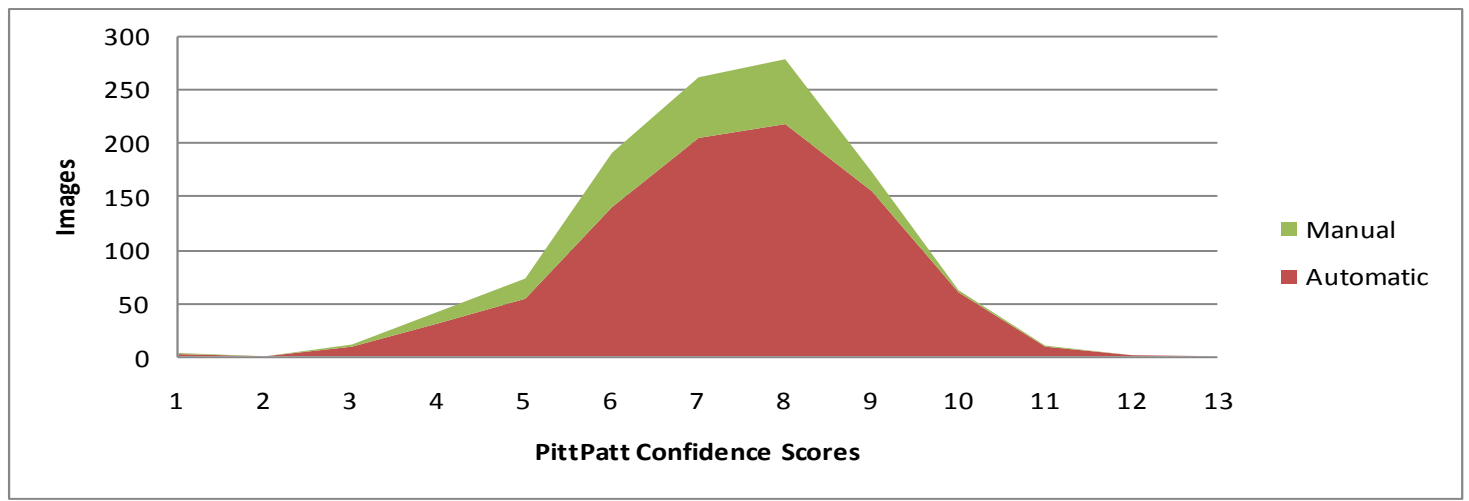

Figure 13 - Correlation of PittPatt Confidence Scores with Automated Landmarking

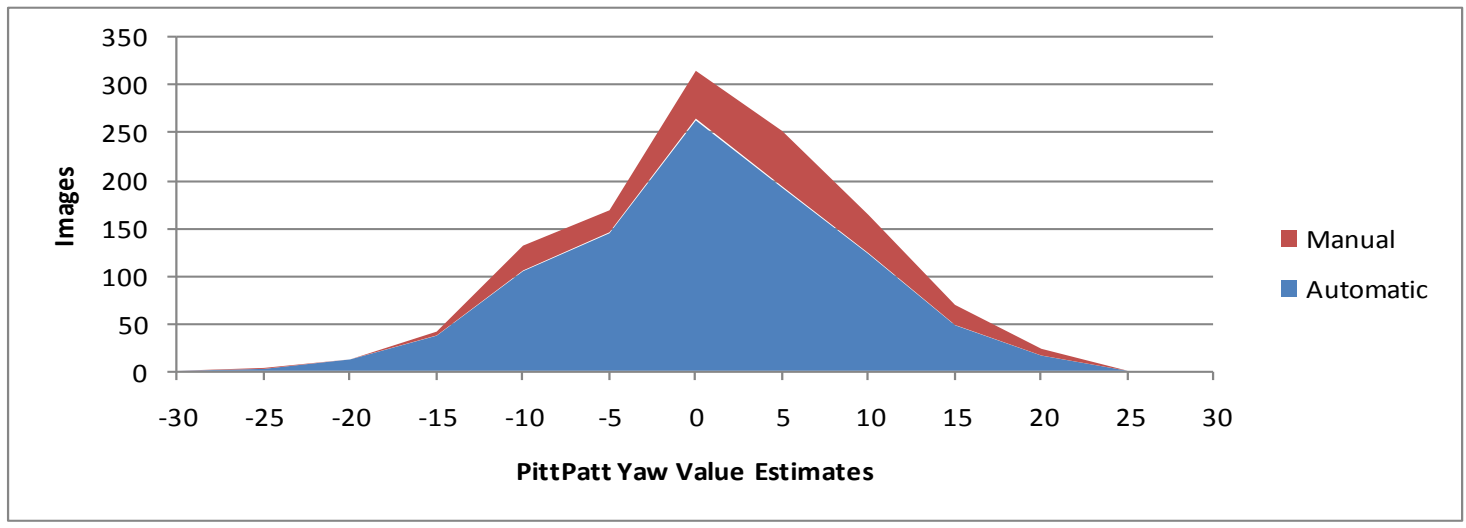

Figure 14 - Correlation of PittPatt Yaw Estimate with Automated Landmarking 


\subsection{Next Steps for Landmarking and the MEDS-II Corpus}

The performance and robustness of facial feature localization is relevant to advancing face recognition and pose conformance, and there are certainly technology advancements yet to be achieved in this area. Additional analysis into the Stasm failures and comparative analysis with other landmarking approaches could be beneficial to recognition systems. Also of interest is to strengthen and better understand the relationships between face morphology (human observable features) and features utilized by machine recognition (i.e., Do they correspond in known ways or are they entirely divergent?).

\section{Face Recognition and Imposters}

Performance of face recognizers depends heavily on the fine tuning of two parameters: the false alarm rate and true acceptance rate. Imposters (i.e. non-mated subjects) are subjects identified in face recognition that are not true subjects, whose match confidence values are larger than the false alarm rate. MITRE performed a study of imposters to identify "look-alikes" to highlight potentially problematic images for face recognizers.

As in the face detection study, MITRE has also used PittPatt to perform face recognition on the images which correspond to the 518 subjects in the corpus. As part of MITRE's experiment, the matcher threshold was set to $0.001 \%$ false acceptance rate to reduce the number of matched subjects in the results. Interestingly, there was a strong correlation between six non-mated subjects. Table 7 tabulates the number of hits on non-mated subjects and displays the images that correspond to imposters.

Table 7 - False Hits

\begin{tabular}{|c|c|c|}
\hline $\begin{array}{c}\text { Query } \\
\text { Subject }\end{array}$ & $\begin{array}{c}\text { Target } \\
\text { Subject }\end{array}$ & $\begin{array}{c}\text { Number } \\
\text { of Hits }\end{array}$ \\
\hline 0388 & 0404 & 5 \\
\hline 0471 & 0396 & 3 \\
\hline 0471 & 0413 & 4 \\
\hline
\end{tabular}

In Table 8 one can id entify a few observations that generally cause problems within face recognition. First, all imposters in the set are comprised of African-American males, even though all subjects in the set were matched against each other. Second, the areas around the orbital region on the face appear to be similar to the human eye. Third, the shape of the nose of all imposters appears to be the same shape. 
Table 8 - Results of Queried Images

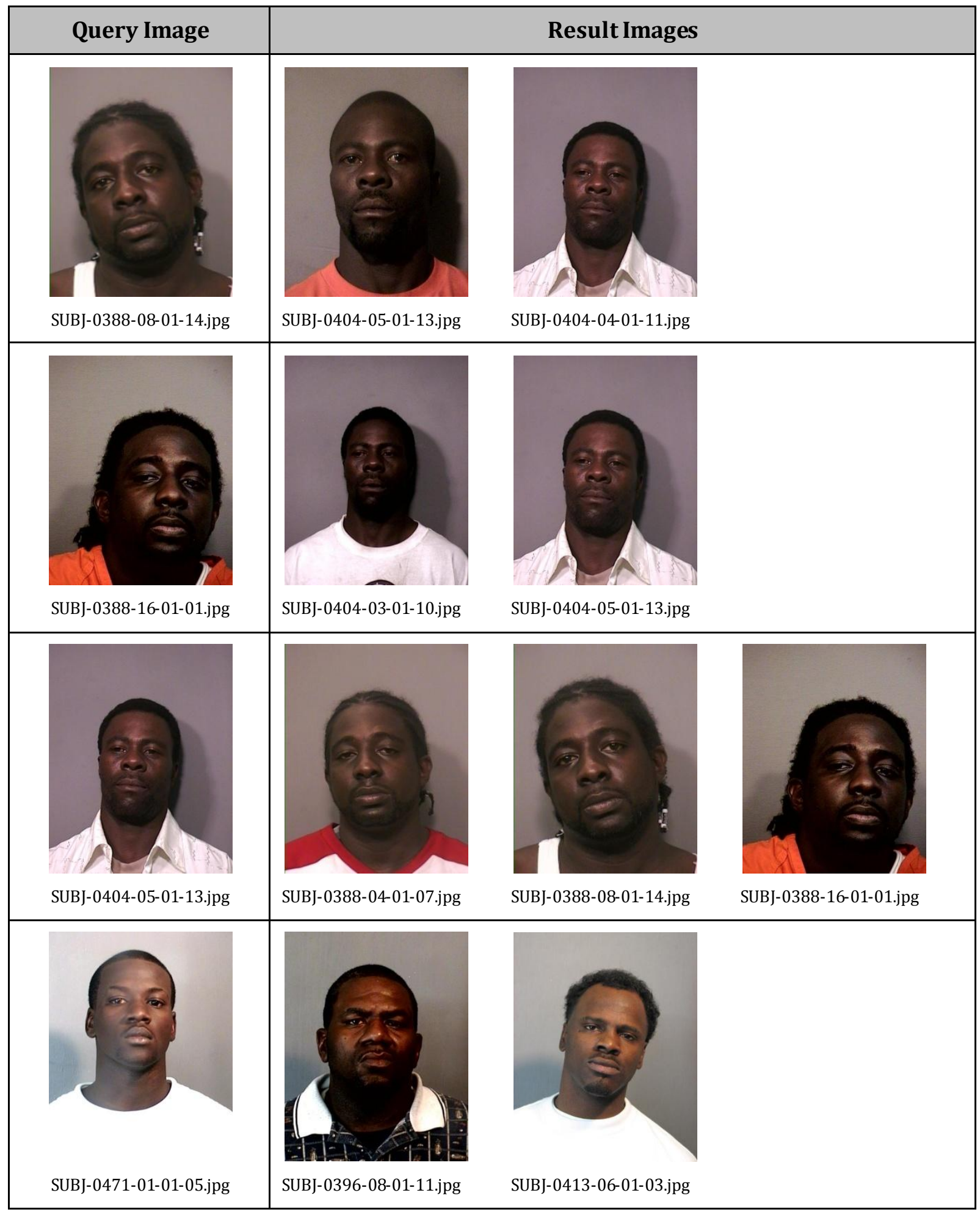


Results of Queried Images Continued

\begin{tabular}{|l|l|l|l|}
\hline Query Image & Result Images \\
\hline SUBJ-0471-03-01-01.jpg & SUBJ-0396-04-01-03.jpg & SUBJ-0396-07-01-05.jpg \\
\hline SUBJ-0413-01-01-08.jpg
\end{tabular}




\section{Appendix A - List of Acronyms}

\begin{tabular}{|l|l|}
\hline \multicolumn{1}{|c|}{ Acronym } & \multicolumn{1}{c|}{ Expansion } \\
\hline ANSI & American National Standards Institute \\
\hline ASM & Active Shape Models \\
\hline BCOE & Biometric Center of Excellence \\
\hline CW & Clockwise \\
\hline CCW & Counter-clockwise \\
\hline COTS & Commercial off the Shelf \\
\hline DOA & Date of Arrest \\
\hline DASL & Data Analysis Support Laboratory \\
\hline EBTS & Electronic Biometric Transmission Specification \\
\hline GOTS & Government off the Shelf \\
\hline ITL & Information Technology Laboratory \\
\hline MEDS & Multiple Encounter Dataset \\
\hline MITRE & The MITRE Corporation \\
\hline NCIC & National Crime Information Center \\
\hline NGI & Next Generation Identification \\
\hline NIST & National Institute of Standards and Technology \\
\hline PHD & Photo Date \\
\hline PSF & Python Software Foundation \\
\hline SMT & Scars, Marks \& Tattoos \\
\hline
\end{tabular}

